\title{
SIMULATION MODELLING OF RESOURCE SHARING IN INTER-ORGANIZATIONAL SUPPLY CHAIN COLLABORATION WITHIN GARMENT INDUSTRY
}

\author{
KE MA $^{123}$, EVA GUSTAFSSON ${ }^{1}$, RUDRAJEET PAL ${ }^{1}$ \\ ${ }^{I}$ Department of Textile management, University of Borås, Sweden; \\ ${ }^{2}$ GEMTEX, ENSAIT, France; \\ ${ }^{3}$ Department of Textile Engineering, Soochow University, China
}

\begin{abstract}
Resource sharing (RS) is an important method in inter-organizational supply chain collaboration (SCC). However, it is still an under-explored area in research of SCC compared to other subjects (e.g. coordinating contract and information sharing). The aim of this paper is to examine the feasibility of applying RS model in the manufacturing stage of garment supply chain and to determine the suitable type of garment for RS in production. Eight scenarios of RS were designed. Discrete-event simulation was used for running experiment of each scenario. The comparison of different scenarios shows that garment manufacturers could get great benefits by applying RS model.
\end{abstract}

\section{Introduction}

Collaboration in supply chain becomes more and more common nowadays; while research regarding supply chain collaboration (SCC) keeps increasing as well. In existing literatures regarding SCC, most of them focused on discussing coordinating contract, information sharing, and joint decision making strategies. In practice, there is an increasing trend of sharing economy in recent years, more and more companies became successful by doing business regarding resource sharing (RS). RS is the process of leveraging capabilities and assets and investing in capabilities and assets with supply chain partners [1]. Partners could share resources (e.g. human or facility resources) if they share same or similar process in e.g. manufacturing or transportation process to improve supply chain performance. RS has been existing in various industries and businesses, e.g. car platform sharing strategy in automobile manufacturing [2], inventory pooling in retailing industry [3] and carriers sharing in logistics [4]. It is not a new concept but is relatively not paid sufficient attention in studies regarding SCC [5].

Studies regarding SCC in garment industry are scarce. Most of them concentrated on coordinating contracts and information sharing by using mathematical modelling, like Xiao and Jin [6], Wang, et al. [7] and Kuo, et al. [8]. To best knowledge, there is no study specifically discussing RS in garment supply chain. Therefore, there is a gap of research regarding RS in garment 
industry. Those successful experiences from other industries imply RS could be a potential new model applying in garment industry so that to improve garment supply chain performance. In practice, current garment manufacturers often desire to keep orders by themselves even if they do not have sufficient resource to finish the order in time under some circumstances. Still, some of them are applying RS within organization itself, resources are shared between different teams or departments for increased productivity and facility utilization. Moreover, RS is applied cross organizations in some garment manufacturers when they are facing special cases, e.g. fluctuation in market expectation or inadequate production capacity. But if this strategy is treated as a common strategy in garment supply chain, RS partners may obtain increased productivity and utilization of facility, resulting in reduced costs and lead time. However, manufacturers who apply RS may incur delay of own production, which could result in a negative effect. Therefore, the objective of this paper is, by building a simulation model of manufacturing stage in garment supply chain and comparing key performance indicator (KPI) of different scenarios in RS model, to examine and analyze whether garment manufacturers can really get benefits by applying $\mathrm{RS}$ and which type of garment is more suitable for this model in production.

\section{Methodology}

\subsection{Conceptual model}

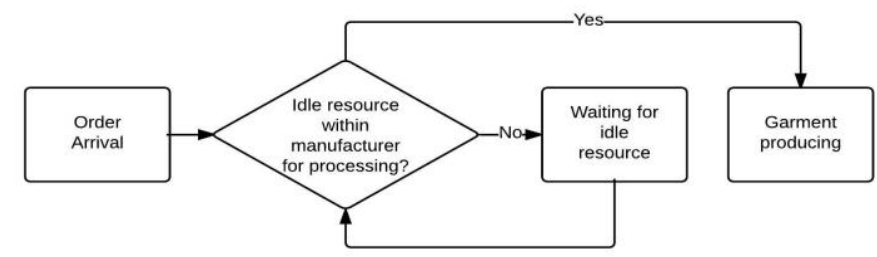

Figure 1. Flowchart of traditional model in garment production

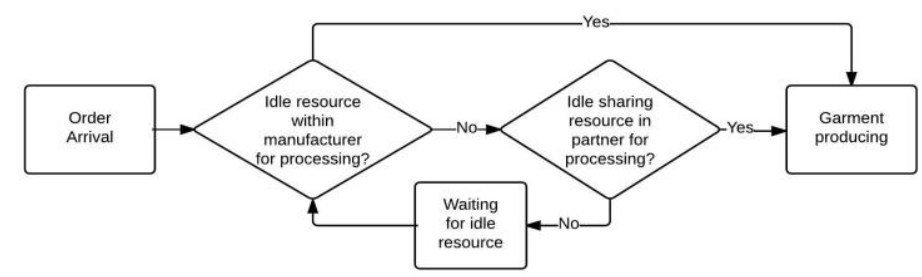

Figure 2. Flowchart of RS model in garment production 
In traditional model of garment production, manufacturers received orders from customers and processed the order based on current resource capacity and production schedule. If there is no idle resource left, then the order should be waited until idle resource is available. The RS model in this study is simple but can make the whole flow and output totally different. If one manufacturer (M1) has no idle resource for processing the order currently, then the resource status of partner (M2) will be checked. The order will be delivered to M2 if it has idle sharing resource for production and no order is waiting for production in M2. A flowchart of the traditional model and the RS model are shown in figure 1 and 2.

\subsection{Simulation model}

As it is almost impossible to run different experiments regarding RS in realworld system, discrete-event simulation (DES) modelling technology was used in this study to evaluate performance of traditional model and different scenarios of RS model. DES, which is one of the most widely used operations research and management techniques, concerns the modeling of a system as it evolves over time by a representation in which the state variables change instantaneously at separate points in time [9].

\subsubsection{DES model building}

The simulation model was built in Simio according to the conceptual model introduced in section 2.1. Two medium-sized garment manufacturers (M1 and M2) with similar production type but different resource capacities were modelled. The simulation model starts with the order arrival to the manufacturers and ends with the finish of garment production. As there are hundreds of types of products in garment production, it is not feasible to build the model on this basis. By analyzing relevant data and considering the objective of this study, garments were categorized based on their order frequency (OF), order size (OS) and production time (PT). PT is directly influenced by the type of garment; therefore three types of PT were defined, viz. long, medium and short. In garment industry, orders are rare to be in high OF with large OS or in low OF with small OS, therefore only the combination of high OF with small OS and low OF with large OS were considered in the DES model. Finally, six typical types of garments (P1-P6) were selected and defined for this model as indicated in table 1. All data were defined based on interviewing professionals in garment manufacturing industry. In the model, there are 40 units of resources (operators and machines) for garment production in M1 and 60 units in M2. To reduce the complexity of the model, the simulation model was developed under following assumptions: 
1. All process in the model followed a First-In-First-Out principle;

2. The influence of raw materials supply was ignored;

3. Breakdown/failure for each process was ignored;

4. Production of each type of garment required same resource in the model;

5. Order was processed immediately if it was processed within the manufacturer; however, a two-hour delay was implemented in RS if the order is sent to partner.

Table 1: Product list and distribution

\begin{tabular}{c|c|c|c|c|c|c}
\hline & P1 & P2 & P3 & P4 & P5 & P6 \\
\hline OF & High & Low & High & Low & High & Low \\
(day) & Un(5,15) & Un(30,50) & Un(5,15) & Un(30,50) & Un(5,15) & Un(30,50) \\
\hline \multirow{2}{*}{ OS } & Small & Large & Small & Large & Small & Large \\
(piece) & Un(50, & Un(3000, & Un(50, & Un(3000, & Un(50, & Un(3000, \\
& $1000)$ & $10000)$ & $1000)$ & $10000)$ & $1000)$ & $10000)$ \\
\hline \multirow{2}{*}{ PT } & Long & Long & Medium & Medium & Short & Short \\
(minute) & Tri(80, & Tri(50, & Tri(35, & Tri(25, 30, & Tri(13, & Tri(8,10, \\
& $90,100)$ & $60,70)$ & $40,45)$ & $35)$ & $15,17)$ & $12)$ \\
\hline
\end{tabular}

* Un = Uniform distribution, Tri = Triangular distribution

\subsubsection{Experiment design and its realization in DES model}

As the initial objective of this study is to explore whether RS is beneficial, a traditional model (scenario 1) and a total RS model (scenario 2) were designed. Another objective of this study is to explore suitable type of garment for applying RS in manufacturing stage. Therefore, another six scenarios (scenario 3-8) were designed, where RS was applied in production of P1-P6 respectively. A complete experiment design is illustrated in table 2.

Table 2: Experiment design

\begin{tabular}{c|c|c|c|c|c|c}
\hline Scenario & RS for P1 & RS for P2 & RS for P3 & RS for P4 & RS for P5 & RS for P6 \\
\hline $\mathbf{1}$ & No & No & No & No & No & No \\
\hline $\mathbf{2}$ & Yes & Yes & Yes & Yes & Yes & Yes \\
\hline $\mathbf{3}$ & Yes & No & No & No & No & No \\
\hline $\mathbf{4}$ & No & Yes & No & No & No & No \\
\hline $\mathbf{5}$ & No & No & Yes & No & No & No \\
\hline $\mathbf{6}$ & No & No & No & Yes & No & No \\
\hline $\mathbf{7}$ & No & No & No & No & Yes & No \\
\hline $\mathbf{8}$ & No & No & No & No & No & Yes \\
\hline
\end{tabular}

In this study, six types of garment have different PT. Therefore, it is not reasonable to compare the total yield $\left(\mathrm{Y}_{\mathrm{T}}\right)$ of six products. In most cases, garment manufacturer can make more profits from products with longer PT. To neutralize the effect of PT, a factor $K_{p}$, which is calculated by the mean PT of 
product $\mathrm{p}$ divided by minimum mean PT among all products, was introduced. Then, a benefit index $(\mathrm{BI})=\sum\left(\mathrm{Y}_{\mathrm{p}} * \mathrm{~K}_{\mathrm{p}}\right)$, performed as KPI to show the potential overall benefit in each scenario, was designed for this study, where $Y_{p}$ is the yield of product $\mathrm{p}$ in two manufacturers.

To realize different RS scenarios in DES model, a general script for programming in Simio is indicated as following:

M1.Capacity.Remaining $==0$ \&\& M2.Capacity.Remaining $>\left(1-\mathrm{R}_{\mathrm{p}}\right) * \mathrm{M} 2$.Capacity $\& \& \sum$ M2.Order.Buffer.NumberWaiting $==0$

$\mathrm{R}_{\mathrm{p}}=0$, Traditional model for production of product $\mathrm{p}$

$\mathrm{R}_{\mathrm{p}}=1$, RS model for production of product $\mathrm{p}$

\section{Results}

Based upon the DES model described in section 2.2.1, eight scenarios were run for duration of 20 weeks with 50 replications respectively. A standard working schedule of five days per week and eight hours per day was employed. An overview of experiment results is shown in table 3 and figure 3.

Table 3: Yield of P1-P6 and Total Yield

\begin{tabular}{c|c|c|c|c|c|c|c|c}
\hline \multicolumn{2}{c|}{ Scenario } & $\mathbf{Y}_{\mathbf{1}}$ & $\mathbf{Y}_{\mathbf{2}}$ & $\mathbf{Y}_{\mathbf{3}}$ & $\mathbf{Y}_{\mathbf{4}}$ & $\mathbf{Y}_{\mathbf{5}}$ & $\mathbf{Y}_{\mathbf{6}}$ & $\mathbf{Y}_{\mathbf{T}}$ \\
\hline \multirow{2}{*}{1} & Mean & 11079.40 & 32570.34 & 10895.80 & 32073.22 & 11385.82 & 34409.70 & 132414.28 \\
\cline { 2 - 9 } & SD & 1472.453 & 3843.222 & 1392.261 & 4943.929 & 1612.821 & 5135.975 & 6497.554 \\
\hline \multirow{2}{*}{2} & Mean & 11050.98 & 34299.76 & 11219.28 & 33092.58 & 11592.10 & 35315.84 & 136570.54 \\
\cline { 2 - 9 } & SD & 1276.691 & 3578.985 & 1646.031 & 5544.957 & 1797.247 & 6390.337 & 6274.972 \\
\hline \multirow{2}{*}{3} & Mean & 11215.78 & 33452.70 & 11339.40 & 33310.94 & 11236.94 & 34636.34 & 135192.10 \\
\cline { 2 - 9 } & SD & 1501.174 & 3564.356 & 1447.861 & 4408.345 & 1716.016 & 6509.319 & 6482.208 \\
\hline \multirow{2}{*}{4} & Mean & 10993.82 & 33286.10 & 10933.88 & 32139.02 & 11359.58 & 33850.36 & 132562.76 \\
\cline { 2 - 9 } & SD & 1397.608 & 3459.663 & 1558.631 & 5027.385 & 1622.016 & 5609.459 & 6918.646 \\
\hline \multirow{2}{*}{5} & Mean & 10833.08 & 33988.90 & 10962.80 & 32685.18 & 11256.08 & 33313.78 & 133039.82 \\
\cline { 2 - 9 } & SD & 1580.126 & 3990.618 & 1600.255 & 4914.563 & 1701.846 & 5284.038 & 5865.334 \\
\hline \multirow{2}{*}{6} & Mean & 11124.98 & 32819.92 & 11055.78 & 33055.54 & 11375.00 & 34403.20 & 133834.42 \\
\cline { 2 - 9 } & SD & 1335.891 & 3705.145 & 1519.647 & 4722.491 & 1711.465 & 6111.260 & 6273.813 \\
\hline 7 & Mean & 10862.26 & 32843.26 & 11246.62 & 33446.22 & 11744.12 & 32357.68 & 132500.16 \\
\cline { 2 - 9 } & SD & 1407.240 & 3921.118 & 1606.443 & 5235.339 & 1819.983 & 6225.637 & 7213.046 \\
\hline \multirow{2}{*}{8} & Mean & 11121.64 & 32665.24 & 11165.46 & 32979.94 & 11474.10 & 35736.64 & 135143.02 \\
\cline { 2 - 8 } & SD & 1444.780 & 3997.147 & 1539.352 & 4651.606 & 1366.536 & 7320.659 & 7251.972 \\
\hline \multirow{2}{*}{} & & & & & & & &
\end{tabular}

Scenario 1 played a benchmark role in the experiment. Firstly, the outcome of scenario 1 and 2 were compared to generally illustrate whether RS have a positive effect on $\mathrm{Y}_{\mathrm{T}}$ and $\mathrm{BI}$ of all six types products. An independent sample TTest (confidence interval $(\mathrm{CI})=95 \%$ ) was conducted to show that there is significant difference in total yield $(\mathrm{t}(98)=3.254, \mathrm{p}=0.002)$ and BI 
$(\mathrm{t}(62.004)=7.344, \mathrm{p}=0.000)$ between scenario 1 and 2 . By comparing the mean value, a $3.14 \%$ increase of $\mathrm{Y}_{\mathrm{T}}$ and a $3.23 \%$ increase of $\mathrm{BI}$ were obtained in scenario 2. It shows that $\mathrm{RS}$, in terms of $\mathrm{Y}_{\mathrm{T}}$ and $\mathrm{BI}$, has an advantage than traditional model. Scenarios 3 to 6 were then respectively compared to scenario 1 to explore the effects when RS applied in different type of products. An increasing (decreasing) percentage of mean value of $\mathrm{Y}_{\mathrm{p}}, \mathrm{Y}_{\mathrm{T}}$ and $\mathrm{BI}$ in each scenario compared to traditional model (scenario 1) is indicated in table 4. Surprisingly, based on the comparison of scenarios 3-8 to 1 , there is no significant difference on $\mathrm{Y}_{\mathrm{p}}$, even on the yield of product applied RS. However, compared to scenario 1, scenario 3, 5, 6 and 8 have significant difference on BI, which means the overall benefit is influenced. Besides, significant effect on $\mathrm{Y}_{\mathrm{T}}$ is obtained in scenario 3 and 8 .

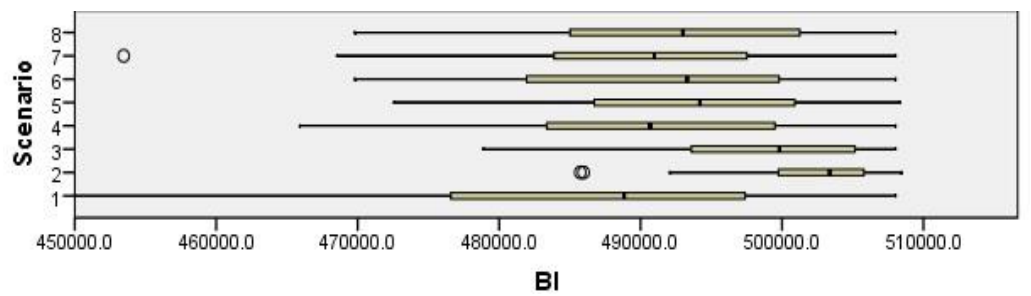

Figure 3. Boxplot of BI

Table 4: Increasing (decreasing) percentage

\begin{tabular}{c|c|c|c|c|c|c|c|c}
\hline Comparison & $\mathbf{Y}_{\mathbf{1}}$ & $\mathbf{Y}_{\mathbf{2}}$ & $\mathbf{Y}_{\mathbf{3}}$ & $\mathbf{Y}_{\mathbf{4}}$ & $\mathbf{Y}_{\mathbf{5}}$ & $\mathbf{Y}_{\mathbf{6}}$ & $\mathbf{Y}_{\mathbf{T}}$ & $\mathbf{B I}$ \\
\hline $\mathbf{2}$ vs.1 & $-0.26 \%$ & $\mathbf{5 . 3 1 \%}$ & $2.97 \%$ & $3.18 \%$ & $1.81 \%$ & $2.63 \%$ & $\mathbf{3 . 1 4 \%}$ & $\mathbf{3 . 2 3 \%}$ \\
\hline $\mathbf{3}$ vs.1 & $1.23 \%$ & $2.71 \%$ & $4.07 \%$ & $3.86 \%$ & $-1.31 \%$ & $0.66 \%$ & $\mathbf{2 . 1 0 \%}$ & $\mathbf{2 . 4 7 \%}$ \\
\hline 4 vs.1 & $-0.77 \%$ & $2.20 \%$ & $0.35 \%$ & $0.21 \%$ & $-0.23 \%$ & $-1.63 \%$ & $0.11 \%$ & $0.67 \%$ \\
\hline $\mathbf{5}$ vs.1 & $-2.22 \%$ & $4.36 \%$ & $0.61 \%$ & $1.91 \%$ & $-1.14 \%$ & $-3.18 \%$ & $0.47 \%$ & $\mathbf{1 . 4 6 \%}$ \\
\hline $\mathbf{6}$ vs.1 & $0.41 \%$ & $0.77 \%$ & $1.47 \%$ & $3.06 \%$ & $-0.10 \%$ & $-0.02 \%$ & $1.07 \%$ & $\mathbf{1 . 1 2 \%}$ \\
\hline 7 vs.1 & $-1.96 \%$ & $0.84 \%$ & $3.22 \%$ & $4.28 \%$ & $3.15 \%$ & $-5.96 \%$ & $0.06 \%$ & $0.76 \%$ \\
\hline $\mathbf{8}$ vs.1 & $0.38 \%$ & $0.29 \%$ & $2.47 \%$ & $2.83 \%$ & $0.78 \%$ & $3.86 \%$ & $\mathbf{2 . 0 6 \%}$ & $\mathbf{1 . 2 8 \%}$ \\
\hline
\end{tabular}

* Bold represents significant difference by independent sample T-Test $(\mathrm{CI}=95 \%, \mathrm{p}<0.05)$

\section{Discussion and conclusion}

This study examined RS model in garment manufacturing stage of interorganizational supply chain collaboration through a simulation model. The experiment results show that RS has a significant effect on increasing total yield and potential benefits of involved manufacturers. An extended investigation reveals that RS has no significant effect on the production of garment type P2 and P5. The total RS model presents the highest increase in overall benefit compared to other scenarios. However, if it is infeasible for manufacturers to 
apply a total RS model, it is recommended to apply RS in production of garment type $\mathrm{P} 1$, as it shows the highest increase in terms of BI. Although the increasing percentage is not sufficiently high, RS model still reveals the potential of bringing great benefits to involved partners, as $1 \%$ increase of BI means approximately 10000 euros profit increase under the condition that $K_{p}=1$ stands for only 2 euros profit. Based on the case in this study, two medium-sized garment manufacturers may obtain a profit increase of over 30000 euros in 20 weeks if a total RS model is applied.

The results of this study also implicates that RS may be applied in other industries which have similar product type and process in production. The simulation in this study was developed on the basis of five assumptions in section 2.2.1; it is expected to develop a more complex and accurate model. There are still several interesting questions to be explored based on the model in this paper, e.g. the optimal combination of products for RS, the effect when more than two manufacturers are involved in RS model, the effect when RS is applied in other stage of supply chain. These are expected to be discussed in future study.

\section{Acknowledgements}

This work is supported by the joint doctorate programme "Sustainable Management and Design for Textiles" which is funded by the European Commission's Erasmus Mundus programme.

\section{References}

1.Cao, M.; Vonderembse, M. A.; Zhang, Q. Y.; Ragu-Nathan, T. S. Int. J. Prod. Res. 2010, 48, (22), 6613-6635.

2.Muffatto, M. Int. J. Prod. Econ. 1999, 60, 145-153.

3.Kurata, H. Int. J. Prod. Res. 2014, 52, (22), 6739-6759.

4.Vilkelis, A.; Jakovlev, S. Transport 2014, 29, (2), 223-230.

5.Ma, K.; Pal, R.; Gustafsson, E. Submitted to Int. J. Prod. Econ. 2015.

6.Xiao, T. J.; Jin, J. A. Prod. Plan. Control 2011, 22, (3), 257-268.

7.Wang, K.; Gou, Q.; Sun, J. W.; Yue, X. H. J. Syst. Sci. Syst. Eng. 2012, 21, (4), 461-479.

8.Kuo, T. C.; Hsu, C. W.; Huang, S. H.; Gong, D. C. Int. J. Comput. Integr. Manuf. 2014, 27, (3), 266-280.

9.Kelton, W. D.; Law, A. M., Simulation modeling and analysis. McGraw Hill Boston: 2000. 